\title{
Investigation of the efficacy and safety of ultrasound- standardized autologous blood injection as treatment for lateral epicondylitis
}

\author{
Christel Braaksma, Jill Otte, Ronald N. Wessel, Nienke Wolterbeek \\ Department of Orthopedic Surgery, St. Antonius Hospital, Nieuwegein, Netherlands
}

Background: There are various conservative treatment options for lateral epicondylitis (LE). The aim is to evaluate pain, daily functioning, and complications after ultrasound-standardized autologous blood injections in patients with LE.

Methods: For this prospective cohort study, consecutive patients (>18 years) diagnosed with LE were included. Autologous blood was injected using a medical device containing an injection disposable with 12 small needles (Instant Tennis Elbow Cure [ITEC]) device. Patient-Rated Tennis Elbow Evaluation (PRTEE), subjective elbow score (SES), palpation and provocation pain, satisfaction, and complications of treatment were measured at baseline and two months after treatment. Paired t-tests and Fisher's exact tests were used for calculating the difference between pre- and post-treatment outcomes.

Results: Fifty-five elbows were analyzed. Mean time between pre- and post-treatment was 11.1 weeks (standard deviation [SD], 8.9 weeks). The mean PRTEE score decreased from 68.2 (SD, 15.7) before surgery to 53.2 (SD, 25.9; $<<0.001$ ) after. The mean SES improved from 36.9 (SD, 20.8) to 51.7 (SD, 27.4; p<0.001). Despite this improvement, only $44.7 \%$ of patients showed relevant clinical improvement in PRTEE, and $37.3 \%$ showed significant clinical improvement based on SES. Four patients reported a complication and the injection disposable failed three times.

Conclusions: Ultrasound-standardized autologous blood injection using the ITEC device is not an effective tool in reducing symptoms related to LE. This study showed that only half of all patients experienced a positive effect. In this heterogeneous cohort of patients, we showed no added value of ultrasound standardization.

Keywords: Tennis elbow; Injection; Conservative treatment; Ultrasound

\section{INTRODUCTION}

Lateral epicondylitis (LE), also known as tennis elbow, is a common tendon disease of the elbow, with a prevalence in the general population between $1 \%$ and $3 \%$. The prevalence is uniformly distributed between men and women and is highest between 45 and 54 years of age $[1,2]$. LE affects the working population who perform repetitive movements of the forearm and/or wrist and is most common in the dominant arm [1-3]. The mean duration of LE complaints is between 6 weeks and 2 years. LE is a self-limiting condition, whereby $83 \%$ of patients recover after one year without treatment [4].

Received: May 31, $2021 \quad$ Revised: November 8, $2021 \quad$ Accepted: November 18, 2021

Correspondence to: Nienke Wolterbeek

Department of Orthopedic Surgery, St. Antonius Hospital, P.O. Box 2500, 3430 EM Nieuwegein, Netherlands

Tel: +31-88-320-2325, E-mail: wolterbeek@antoniusziekenhu is.nl, ORCID: https://orcid.org/0000-0003-1745-1417

\section{Financial support: None.}

Conflict of interest: None.

Copyright@C 2022 Korean Shoulder and Elbow Society.

This is an Open Access article distributed under the terms of the Creative Commons Attribution Non-Commercial License (http://creativecommons.org/licenses/by-nc/4.0/) which permits unrestricted non-commercial use, distribution, and reproduction in any medium, provided the original work is properly cited. 
Pain in the lateral elbow is the most common symptom of LE and is most often caused by chronic overuse and subsequent degeneration of the involved tendon $[1,5,6]$. The origin of the extensor carpi radialis brevis (ECRB) tendon, with attachment 2 $\mathrm{cm}$ distal from the lateral epicondyle, is implicated in $90 \%$ of all LE cases [5].

There is no established optimal treatment for LE. Treatment varies from injections with corticosteroid, platelet-rich plasma (PRP), or autologous blood; physical therapy; non-steroidal anti-inflammatory drugs (NSAIDs); immobilization splints or casts; and/or surgery. Previous meta-analyses and studies have shown that PRP, corticosteroids, and autologous blood injections can have a positive effect on pain and function. However, results are not consistent, and the degree of reported effect varies greatly among studies [7-14]. A recent meta-analysis has shown with moderate certainty that autologous blood or PRP injection probably provides little or no clinically important benefit for pain or function compared with placebo injection [8]. In most injection studies, injections were performed without ultrasound or other guidance. Several studies have shown lower accuracy of placement of unguided injections compared to ultrasound-guided injections [11,15-20]. Despite the unclear and perhaps disappointing treatment results and the self-limiting condition of LE, physicians are attempting to shorten the duration of symptoms and improve functionality in patients with LE. Because ultrasound-guided injections are not always possible in daily practice, we studied an administration method that lies between ultrasound-guided and free-hand injections.

Here, we evaluated pain, daily functioning, and possible complications after ultrasound-standardized autologous blood injections in patients with LE. The hypothesis was that patients treated with ultrasound-standardized autologous blood injection would experience pain relief and improvement in daily functioning within two months.

\section{METHODS}

This study is approved by Institutional Review Board of the St. Antonius Hospital (IRB No. Z17.019). Written informed consent was obtained from the patients.

For this observational prospective cohort study, all consecutive patients diagnosed with LE referred for ultrasound-standardized injections with autologous blood between December 2016 and December 2017 were eligible for inclusion. Data were collected using REDCap (Research Electronic Data Capture), a secure, web-based, electronic data capture tool for research studies [21].

The inclusion criteria were patients diagnosed with LE in which conservative treatment failed and point tenderness was noted during palpation over the common extensor origin and provocation pain of the lateral epicondyle, tested by resisted dorsiflexion of the wrist with a pronated forearm and extended elbow. Exclusion criteria were age younger than 18 years and other elbow pain or injuries such as medial epicondylitis, local arthritis, arthrosis, synovitis, ganglion cyst, plica radio-humeral, impingement, or posterior interosseous nerve entrapment. It was decided in advance to include patients with previous cortisone injections, concomitant neck pain, and/or fibromyalgia. In this way, despite the risk of heterogeneity, a larger population that most closely resembled the population encountered in everyday orthopedic practice was eligible for inclusion.

\section{Treatment}

All patients underwent ultrasound-standardized autologous blood injection. The skin was first anesthetized with $3 \mathrm{~mL}$ lidocaine in a sterile condition. Subsequently, the depth and location of the ECRB were measured by ultrasound (Philips CX50, Eindhoven, the Netherlands). During the ultrasound, the patient sat in a relaxed position, with the arm resting on a table at shoulder height and an elbow flexion of $90^{\circ}$. Depth was defined on screenshots of the ultrasound by placing one marker at the middle of the tendon and the other marker at the skin. The intended location was marked on the skin with a cross.

From the unaffected arm, $1 \mathrm{~mL}$ blood was obtained by venous puncture. This autologous blood was reinjected within 5 minutes in the affected elbow using an Instant Tennis Elbow Cure (ITEC; ITEC Medical BV, Enschede, the Netherlands) device containing an injection disposable with 12 small needles. This device was developed to support standardized perforation using laser alignment and depth regulation. The 12 needles were arranged in a $3 \times 4$ formation and adapted to the anatomy of the ECRB. The depth of the needles was set to match the ultrasound measured depth. After the skin was washed with alcohol, the 12 needles were positioned above the ultrasound-determined location and were pushed down to perforate the skin and tendon (Fig. 1). During the perforation, $0.5 \mathrm{~mL}$ autologous blood was injected into the tendon via the needles. Subsequently, the needles were lifted, the injection disposable removed, and the injection site was covered with an adhesive plaster.

Patients were informed about stiffness of the treated elbow. Rest was recommended; however, there were no restrictions on movement other than symptom-causing activities. Any complications during or directly after treatment were recorded. All patients were treated by the same dedicated physician, trained in performing this treatment. 


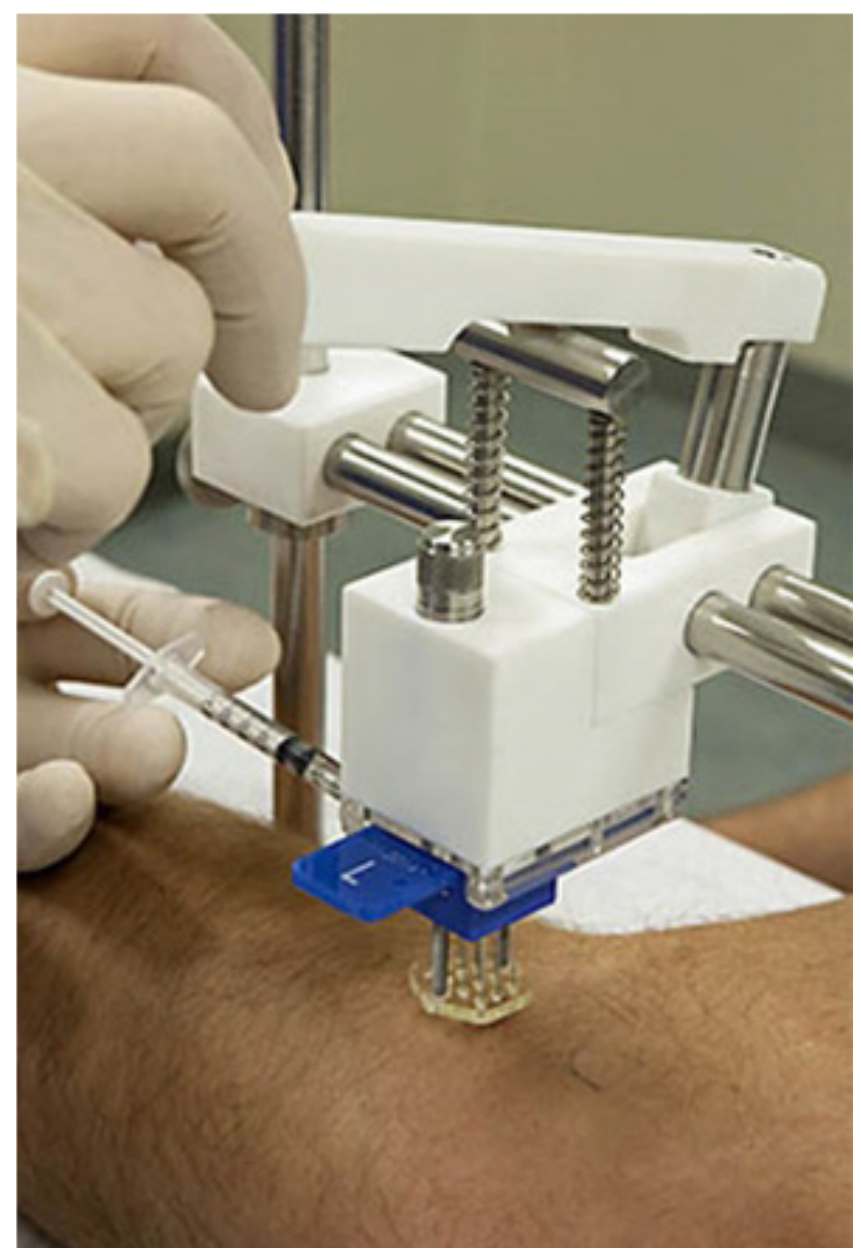

Fig. 1. Image showing the medical device containing the injection disposable with 12 small needles in blue. The depth of the needles was set to match the ultrasound measured depth. The needles are pushed down with a handle. During perforation, autologous blood is injected into the tendon via the needles. Subsequently, the needles are lifted, and the injection disposable is removed.

\section{Outcome Measures}

Outcomes were measured at baseline and two months after treatment. The primary outcome was the Patient-Rated Tennis Elbow Evaluation (PRTEE) [22]. Secondary outcomes were subjective elbow score (SES) [23], palpation and provocation pain, satisfaction of treatment (four items), and complications of treatment. Baseline characteristics of age, sex, dominant arm, duration and possible cause of complaints, previous elbow treatment, concomitant neck pain, and fibromyalgia were also recorded.

The PRTEE is a validated questionnaire that assesses pain and functionality of the affected elbow during the previous week on a scale of $0-10$. The questionnaire contains 15 questions including five about pain and 10 about functionality. When a patient is not able to perform an activity because of elbow pain, the item should be scored as "10," as higher scores indicate greater pain and disability [22]. The minimal detectable change of the PRTEE was previously determined in a Dutch study at 9 points; therefore, clinically relevant improvement change was set at $\geq 11$ points [22].

The SES is a single item self-completed measure in which patients are asked to grade their elbow as a percentage of a normal elbow (100\%). The minimal clinical important difference of this value is unknown; however, based on the literature, a change of 20 points was adopted as an obvious change in outcome [23].

Pain during palpation and provocation pain were measured using a 4-point Likert subscale with the following scores: none, mild, moderate, or severe pain. Satisfaction was measured using four items with a 7-point Likert scale: (1) How has your overall daily functioning changed since the treatment on your elbow? (2) The pain around my elbow is reduced by the treatment. (3) I am satisfied with the result of the treatment. (4) If I need treatment again, I would choose this treatment again. The answer options varied between "very much deteriorated" and "very much improved" to "totally disagree" and "totally agree."

\section{Statistical Analysis}

Data were analyzed using IBM SPSS ver. 24 (IBM Corp., Armonk, NY, USA). Paired t-tests were used for calculating significant differences between the PRTEE score and SES pre- and post-treatment. Fisher's exact tests were used for determining the differences in provocation and palpation pain pre- and post-treatment. A p-value $<0.05$ was considered significant. Linear regression analysis was used to determine the influence of concomitant neck pain and fibromyalgia on the PRTEE outcome score.

\section{RESULTS}

During the inclusion period, the treatment was performed in 65 unique patients. Ten patients were, despite our effort, lost to follow-up for the post-treatment evaluation and excluded from analyses (Fig. 2). The mean time between the pre- and post-treatment was 11.1 weeks (standard deviation [SD], 8.9 weeks) (Table 1).

\section{Complications}

Four patients reported a complication after treatment including spontaneous hematoma, severe pain and tightness around the elbow, severe pain and a swollen arm, and numb feeling in the middle finger. None of the patients received additional treatment in response to the complication. In addition, the treating physician experienced a few complications with the performance of the device as the injection disposable failed three times, once each in three patients. In all three instances, the injection dispos- 
able was blocked while pushed down and needed to be replaced before skin perforation.

\section{Patient-Rated Tennis Elbow Evaluation}

The mean pre- and post-treatment PRTEE scores are presented in Table 2. The mean improvement in total score was a decrease of 14.7 (SD, 22.5) points on a $0-100$ scale. At follow-up, $44.7 \%$ of the patients showed relevant clinical improvement and $8.5 \%$ showed relevant clinical deterioration (Fig. 3). Linear regression analysis showed that concomitant neck pain and fibromyalgia did not influence the PRTEE outcome score.

\section{Secondary Outcomes}

The mean SES pre-treatment and post-treatment scores are presented in Table 2. The mean improvement was 14.6 points on a $0-100$ scale, which was below the a priori defined threshold of 20

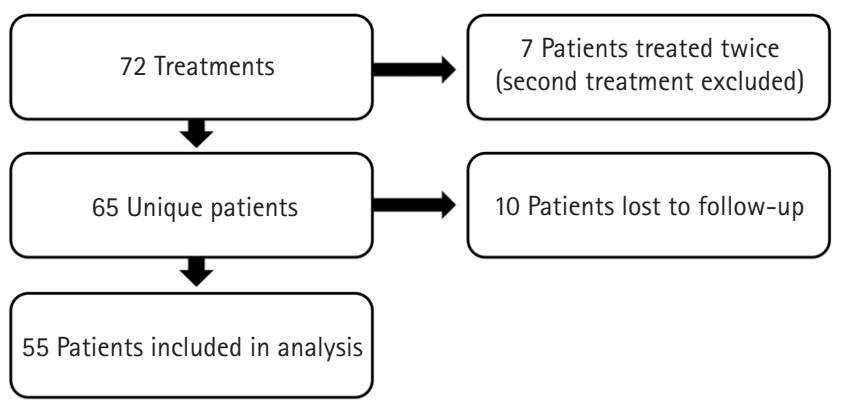

Fig. 2. Flowchart of the included patients.
Table 1. Patient demographics

\begin{tabular}{|c|c|}
\hline Variable & $\begin{array}{l}\text { Included in analysis } \\
\qquad(\mathrm{n}=55)\end{array}$ \\
\hline Age (yr) & $48.8 \pm 9.4(35-88)$ \\
\hline \multicolumn{2}{|l|}{ Sex } \\
\hline Female & $35(63.6)$ \\
\hline Male & $20(36.4)$ \\
\hline \multicolumn{2}{|l|}{ Dominant arm } \\
\hline Right & $50(90.9)$ \\
\hline Left & $5(9.1)$ \\
\hline \multicolumn{2}{|l|}{ Affected arm } \\
\hline Right & $38(69.1)$ \\
\hline Left & $17(30.9)$ \\
\hline \multicolumn{2}{|l|}{ Fibromyalgia } \\
\hline Yes & $5(9.1)$ \\
\hline No & $50(90.9)$ \\
\hline \multicolumn{2}{|l|}{ Concomitant neck pain } \\
\hline Yes & $15(27.3)$ \\
\hline No & $31(56.4)$ \\
\hline Unknown & $9(16.4)$ \\
\hline \multicolumn{2}{|l|}{ Duration of complaints } \\
\hline$<6 \mathrm{mo}$ & $4(7.3)$ \\
\hline $6-12 \mathrm{mo}$ & $16(29.1)$ \\
\hline $1-2 \mathrm{yr}$ & $24(43.6)$ \\
\hline$>2 \mathrm{yr}$ & $11(20.0)$ \\
\hline \multicolumn{2}{|l|}{ Cause of complaints } \\
\hline Unclear & $28(50.9)$ \\
\hline Trauma & $4(7.3)$ \\
\hline Overuse & $23(41.8)$ \\
\hline Time between pre- and post-treatment (wk) & $11.1 \pm 8.9(4-54)$ \\
\hline
\end{tabular}

Values are presented as mean \pm standard deviation (range) or number (\%).

Table 2. Primary and secondary outcome measures $(n=55)$

\begin{tabular}{|c|c|c|c|}
\hline Variable & Pre-treatment & Post-treatment & p-value \\
\hline \multicolumn{4}{|l|}{ PRTEE } \\
\hline Subdomain pain $(0-50)$ & $34.6 \pm 7.0$ & $26.7 \pm 12.4$ & $<0.001$ \\
\hline Subdomain function $(0-50)$ & $33.6 \pm 10.3$ & $26.5 \pm 14.3$ & 0.001 \\
\hline Total score $(0-100)$ & $68.2 \pm 15.7$ & $53.2 \pm 25.9$ & $<0.001$ \\
\hline Subjective elbow score $(0-100)$ & $36.9 \pm 20.8$ & $51.7 \pm 27.4$ & $<0.001$ \\
\hline Palpation pain & & & $<0.001$ \\
\hline None & $1(1.8)$ & $7(12.7)$ & \\
\hline Mild & $7(12.7)$ & $18(32.7)$ & \\
\hline Moderate & $24(43.6)$ & $9(16.4)$ & \\
\hline Severe & $23(41.8)$ & $18(32.7)$ & \\
\hline Missing & - & $3(5.5)$ & \\
\hline Provocation pain & & & $<0.001$ \\
\hline None & 0 & $13(23.6)$ & \\
\hline Mild & $11(20.0)$ & $16(29.1)$ & \\
\hline Moderate & $23(41.8)$ & $10(18.2)$ & \\
\hline Severe & $21(38.2)$ & $9(16.4)$ & \\
\hline Missing & - & $7(12.7)$ & \\
\hline
\end{tabular}

Values are presented as mean \pm standard deviation or number (\%).

PRTEE: patient-rated tennis elbow evaluation. 


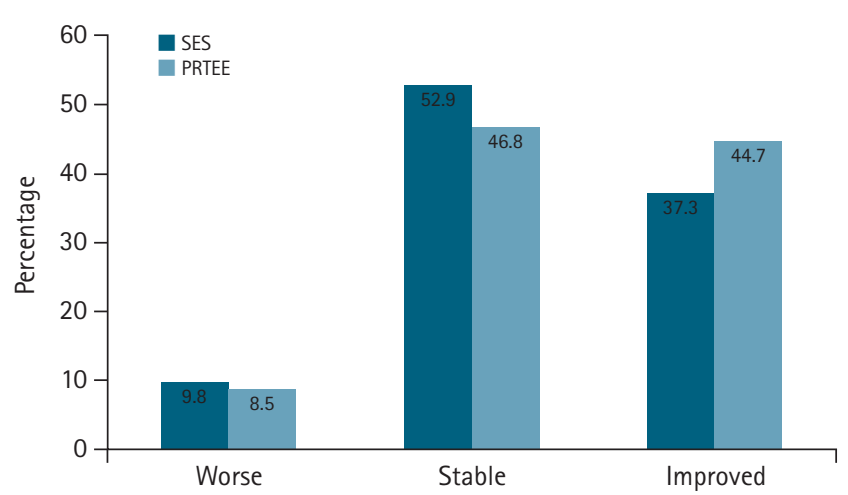

Fig. 3. Changes in patient-rated tennis elbow evaluation (PRTEE) and subjective elbow score (SES) divided into three categories: worse, stable, and improved. A worse PRTEE change score was defined as an increase $>12$ points, stable as a change score between -11 and 11 points, and improved as a change score $<12$ points. A worse SES was defined as a decrease $>20$ points, stable as a change score between -19 and 19 points, and improved as a change score $>20$ points. points. With this threshold, $9.8 \%$ of the patients were classified with a worse condition, and only $37.3 \%$ were classified as improved post-treatment Fig. 3. Both palpation and provocation pain were significantly different after treatment $(\mathrm{p}<0.001)$. Four questions were asked of each patient to determine satisfaction with treatment (Table 3). Concerning function, 56.4\% indicated slightly to very much improved. Pain was reduced in $45.4 \%$ of patients, and $45.4 \%$ were satisfied with the treatment, while $58.2 \%$ of patients would choose this treatment again.

\section{DISCUSSION}

Here, we evaluated pain, daily functioning, and possible complications using the ITEC device in patients with LE. The mean improvement in PRTEE total score was a decrease of 14.7 points on a $0-100$ scale, which was larger than the clinically relevant im-

Table 3. Results of satisfaction

\begin{tabular}{|c|c|c|c|}
\hline Item & Answer option & Percentage & $\begin{array}{l}\text { Percentage positive, } \\
\text { neutral, or negative }\end{array}$ \\
\hline \multirow{7}{*}{$\begin{array}{l}\text { How has your overall daily functioning changed since the treatment } \\
\text { on your elbow? }\end{array}$} & Very much deteriorated & 1.8 & 9.1 \\
\hline & Strongly deteriorated & 7.3 & \\
\hline & Slightly deteriorated & 0 & \\
\hline & Neither improved nor deteriorated & 30.9 & 30.9 \\
\hline & Slightly improved & 23.6 & 56.4 \\
\hline & Strongly improved & 16.4 & \\
\hline & Very much improved & 16.4 & \\
\hline \multirow[t]{7}{*}{ The pain around my elbow was reduced by the treatment. } & Totally disagree & 12.7 & 32.7 \\
\hline & Strongly disagree & 5.5 & \\
\hline & Slightly disagree & 14.5 & \\
\hline & Neither agree nor disagree & 18.2 & 18.2 \\
\hline & Slightly agree & 16.4 & 45.4 \\
\hline & Strongly agree & 14.5 & \\
\hline & Totally agree & 14.5 & \\
\hline \multirow[t]{7}{*}{ I am satisfied with the result of the treatment. } & Totally disagree & 20.0 & 41.9 \\
\hline & Strongly disagree & 5.5 & \\
\hline & Slightly disagree & 16.4 & \\
\hline & Neither agree nor disagree & 9.1 & 9.1 \\
\hline & Slightly agree & 12.7 & 45.4 \\
\hline & Strongly agree & 10.9 & \\
\hline & Totally agree & 21.8 & \\
\hline \multirow[t]{7}{*}{ If I need treatment again, I will choose this treatment again. } & Totally disagree & 16.4 & 25.5 \\
\hline & Strongly disagree & 3.6 & \\
\hline & Slightly disagree & 5.5 & \\
\hline & Neither agree nor disagree & 12.7 & 12.7 \\
\hline & Slightly agree & 18.2 & 58.2 \\
\hline & Strongly agree & 5.5 & \\
\hline & Totally agree & 34.5 & \\
\hline
\end{tabular}


provement change of 11 points. Despite this, $46.8 \%$ of the patients were stable and $8.5 \%$ showed deterioration. Therefore, the mean improvement of 14.7 points represented less than half of the patients. The same was seen in two of the four satisfaction items, where only $45.4 \%$ of the patients were positive about pain reduction or satisfied with the treatment. Concerning change in functional improvement, a small majority was positive (56.4\%) compared to $30.9 \%$ that indicated no improvement or deterioration and $9.1 \%$ that indicated deterioration. When asking if patients would opt for this treatment again, 58.2\% indicated that they would and $25.5 \%$ indicated that they would not. This percentage is higher than the percentage of patients that were satisfied. This might be explained by the hope that they will benefit from a second treatment. The SES showed a significant improvement of 14.6 points on a $0-100$ scale. This change was below the defined threshold of 20 points. The minimal clinically relevant change for this score is not yet determined and, therefore, this threshold might be too high. With this threshold, only $37.3 \%$ of patients indicated improvement post-treatment.

The general impression of this cohort study was that only half of the patients experienced a positive effect of the ultrasound-standardized autologous blood injections. This is in accordance with previous results of other non-operative treatments such as physical therapy; bracing; NSAIDs; injections with cortisone, botulinum toxin, or autologous blood; and/or PRP [6,10-12,24]. A placebo effect and the self-limiting course of the illness might have played a role in this improvement.

The literature has shown lower accuracy of unguided injections compared to ultrasound-guided injections [15-20]. The lower accuracy might result in suboptimal treatment outcomes. The ultrasound-standardized method used in this study partly resolves this issue. With this method, ultrasound was used to determine the optimal location and depth of the injection before administration. However, during injection, ultrasound was not used and there was no certainty about the accuracy of the location. A large area was injected because of the injection disposable with 12 needles. This might result in a margin of error regarding location, which might benefit the accuracy.

Patients in this study showed some complications after treatment. Skin reaction and local injection site pain are known side effects of autologous blood injections [9]. Furthermore, in a clinical setting, physicians need to be able to rely on the device, which was not always the case in this study. With three patients, the injection disposable was dysfunctional and needed to be replaced during treatment. This involved extra time and cost.

There are several limitations of this study. The primary limitation is that there was no control group. It was a deliberate deci- sion to first perform a cohort study, instead of a randomized controlled trial, to assess the feasibility and practicability of the ultrasound-standardized method in our clinic. After this cohort study, we are not convinced of the added value of this ultrasound-standardized injection method. Randomized controlled trials with a longer follow-up are needed to provide a definite answer about the added value of ultrasound-standardized injections in patients with LE compared to free hand injections. As mentioned before, with the current method, ultrasound was used to determine the optimal location and depth of the injection before the injection was given. The lack of ultrasound guidance during the injection is, in our opinion, a major limitation of this device as there is no certainty about the depth and accuracy of the location.

The included cohort was heterogeneous, most closely resembling the normal LE population encountered in everyday orthopedic practice. It could be that previous cortisone injections might have interfered with the outcomes. Nevertheless, in this study, concomitant neck pain and fibromyalgia did not influence the PRTEE outcome score. In future studies, these factors might be considered as exclusion criteria to create a more homogeneous study group [25].

The wide range (4-54 weeks) between follow-up visits was also a limitation. Patients with LE are in general an unreliable patient group regarding visit and therapy compliance. Some patients that showed positive progress were reluctant to make an appointment for the post-treatment visit. On the other hand, patients with disappointing results did not want to wait eight weeks for additional treatment and came back earlier than the predetermined follow-up window or did not want to come back at all. This explains the high loss of patients to follow-up. In future studies, it is recommended to maintain the follow-up window more strictly, especially because LE is a self-limiting condition usually resolving over a 12 - to 18 -month period without treatment [24].

The last limitation concerns the PRTEE score. Several patients did not respond to items about sport and work or answered the questions incorrectly. Instructions were to score an item "10" (worse score) when it could not be performed because of elbow pain and to leave the item blank when the item was not performed. However, some patients left items blank when "10" should have been answered, and some answered " 0 " (no problem or pain) for incorrect items. Most of these issues were resolved immediately by the physician. Nonetheless, when using the PRTEE in research or clinical setting, it should be kept in mind that patients might not be able to fill out this questionnaire independently. 
Ultrasound-standardized autologous blood injection using the ITEC device is not an effective tool in reducing symptoms related to LE. This study shows that only half of the patients experienced a positive effect. This heterogeneous cohort of patients showed no added value of ultrasound-standardized injections.

\section{ORCID}

Nienke Wolterbeek https://orcid.org/0000-0003-1745-1417

\section{REFERENCES}

1. Shiri R, Viikari-Juntura E, Varonen H, Heliövaara M. Prevalence and determinants of lateral and medial epicondylitis: a population study. Am J Epidemiol 2006;164:1065-74.

2. Walker-Bone K, Palmer KT, Reading I, Coggon D, Cooper C. Prevalence and impact of musculoskeletal disorders of the upper limb in the general population. Arthritis Rheum 2004;51: 642-51.

3. Shiri R, Varonen H, Heliövaara M, Viikari-Juntura E. Hand dominance in upper extremity musculoskeletal disorders. J Rheumatol 2007;34:1076-82.

4. Smidt N, van der Windt DA. Tennis elbow in primary care. BMJ 2006;333:927-8.

5. Bhabra G, Wang A, Ebert JR, Edwards P, Zheng M, Zheng MH. Lateral elbow tendinopathy: development of a pathophysiology-based treatment algorithm. Orthop J Sports Med 2016;4: 2325967116670635.

6. Shiri R, Viikari-Juntura E. Lateral and medial epicondylitis: role of occupational factors. Best Pract Res Clin Rheumatol 2011; 25:43-57.

7. Karjalainen TV, Silagy M, O'Bryan E, Johnston RV, Cyril S, Buchbinder R. Autologous blood and platelet-rich plasma injection therapy for lateral elbow pain. Cochrane Database Syst Rev 2021;9:CD010951.

8. Linnanmäki L, Kanto K, Karjalainen T, Leppänen OV, Lehtinen J. Platelet-rich plasma or autologous blood do not reduce pain or improve function in patients with lateral epicondylitis: a randomized controlled trial. Clin Orthop Relat Res 2020;478:1892900.

9. Arirachakaran A, Sukthuayat A, Sisayanarane T, Laoratanavoraphong S, Kanchanatawan W, Kongtharvonskul J. Platelet-rich plasma versus autologous blood versus steroid injection in lateral epicondylitis: systematic review and network meta-analysis. J Orthop Traumatol 2016;1:101-12.

10. Coombes BK, Bisset L, Vicenzino B. Efficacy and safety of corticosteroid injections and other injections for management of tendinopathy: a systematic review of randomised controlled trials. Lancet 2010;376:1751-67.

11. Krogh TP, Bartels EM, Ellingsen T, et al. Comparative effectiveness of injection therapies in lateral epicondylitis: a systematic review and network meta-analysis of randomized controlled trials. Am J Sports Med 2013;41:1435-46.

12. Mishra AK, Skrepnik NV, Edwards SG, et al. Efficacy of platelet-rich plasma for chronic tennis elbow: a double-blind, prospective, multicenter, randomized controlled trial of $230 \mathrm{pa}-$ tients. Am J Sports Med 2014;42:463-71.

13. Sussman WI, Williams CJ, Mautner K. Ultrasound-guided elbow procedures. Phys Med Rehabil Clin N Am 2016;27:573-87.

14. Thanasas C, Papadimitriou G, Charalambidis C, Paraskevopoulos I, Papanikolaou A. Platelet-rich plasma versus autologous whole blood for the treatment of chronic lateral elbow epicondylitis: a randomized controlled clinical trial. Am J Sports Med 2011;39:2130-4.

15. Borbas P, Kraus T, Clement H, Grechenig S, Weinberg AM, Heidari N. The influence of ultrasound guidance in the rate of success of acromioclavicular joint injection: an experimental study on human cadavers. J Shoulder Elbow Surg 2012;21:16947.

16. Cunnington J, Marshall N, Hide G, et al. A randomized, double-blind, controlled study of ultrasound-guided corticosteroid injection into the joint of patients with inflammatory arthritis. Arthritis Rheum 2010;62:1862-9.

17. Finnoff JT, Nutz DJ, Henning PT, Hollman JH, Smith J. Accuracy of ultrasound-guided versus unguided pes anserinus bursa injections. PM R 2010;2:732-9.

18. Hashiuchi T, Sakurai G, Morimoto M, Komei T, Takakura Y, Tanaka Y. Accuracy of the biceps tendon sheath injection: ultrasound-guided or unguided injection? A randomized controlled trial. J Shoulder Elbow Surg 2011;20:1069-73.

19. Kim TK, Lee JH, Park KD, Lee SC, Ahn J, Park Y. Ultrasound versus palpation guidance for intra-articular injections in patients with degenerative osteoarthritis of the elbow. J Clin Ultrasound 2013;41:479-85.

20. Muir JJ, Curtiss HM, Hollman J, Smith J, Finnoff JT. The accuracy of ultrasound-guided and palpation-guided peroneal tendon sheath injections. Am J Phys Med Rehabil 2011;90:564-71.

21. Harris PA, Taylor R, Thielke R, Payne J, Gonzalez N, Conde JG. Research electronic data capture (REDCap): a metadata-driven methodology and workflow process for providing translational research informatics support. J Biomed Inform 2009;42:377-81.

22. van Ark M, Zwerver J, Diercks RL, van den Akker-Scheek I. Cross-cultural adaptation and reliability and validity of the Dutch Patient-Rated Tennis Elbow Evaluation (PRTEE-D). 
BMC Musculoskelet Disord 2014;15:270.

23. Schneeberger AG, Kösters MC, Steens W. Comparison of the subjective elbow value and the Mayo elbow performance score. J Shoulder Elbow Surg 2014;23:308-12.

24. Sims SE, Miller K, Elfar JC, Hammert WC. Non-surgical treatment of lateral epicondylitis: a systematic review of randomized controlled trials. Hand (N Y) 2014;9:419-46.

25. Smidt N, Lewis M, VAN DER Windt DA, Hay EM, Bouter LM, Croft P. Lateral epicondylitis in general practice: course and prognostic indicators of outcome. J Rheumatol 2006;33:205359. 